\title{
Matrix averages relating to the Ginibre ensembles
}

\author{
Peter J. Forrester* and Eric M. Rains ${ }^{\dagger}$ \\ *Department of Mathematics and Statistics, University of Melbourne, \\ Victoria 3010, Australia ; \\ $\dagger$ Department of Mathematics, California Institute of Technology, Pasadena, CA 91125, USA \\ The theory of zonal polynomials is used to compute the average of a Schur polynomial of \\ argument $A X$, where $A$ is a fixed matrix and $X$ is from the real Ginibre ensemble. This \\ generalizes a recent result of Sommers and Khorozhenko [J. Phys. A 42 (2009), 222002], \\ and furthermore allows analogous results to be obtained for the complex and real quaternion \\ Ginibre ensembles. As applications, the positive integer moments of the general variance \\ Ginibre ensembles are computed in terms of generalized hypergeometric functions, these are \\ written in terms of averages over matrices of the same size as the moment to give duality \\ formulas, and the averages of the power sums of the eigenvalues are expressed as finite sums \\ of zonal polynomials.
}

\section{Introduction}

The statistical properties of the eigenvalues of random $N \times N$ matrices with independent, identically distributed (i.i.d.) real entries is a prominent topic in both physics and mathematics. Physical applications began with the paper of May [23] on the stability of an ecological network consisting of many components $\left\{y_{i}(t)\right\}_{i=1, \ldots, N}$, coupled in some unknown way. Suppose the evolution of the components is governed by a first order differential system, and write the linearization of the latter about a fixed point in the form

$$
\left[\frac{d \tilde{y}_{i}(t)}{d t}\right]_{i=1, \ldots, N}=\left(-\mathbb{I}_{N}+B\right)\left[\tilde{y}_{i}(t)\right]_{i=1, \ldots, N} .
$$

With the $N \times N$ matrix $B$ having all entries zero this differential equation exhibits exponential relaxation to the fixed point. For general $B$ the system (1.1) is stable if and only if all the eigenvalues of $B$ have real part less than or equal to 1 .

Since the coupling between components is unknown, it is reasonable to take the components of $B$ to be random. May argued that for $B$ a dilute matrix (fraction $1-c$ of its elements zero) with non-zero elements i.i.d. random variables having mean zero and variance $\sigma^{2}$, the spectral radius will be less than 1 provided $\sigma \sqrt{N c}<1$. This is an asympototic result, requiring that $N$ be large. It is consistent with a rigorous result proved subsequently for the special case $c=1$ [15].

In the mathematics literature, attention has focussed not only on the spectral radius, but also the eigenvalue density. With the elements standard Gaussians, by explicit calculation the eigenvalue density was proved to be asymptotically uniform in the disk of radius $\sqrt{N}$, centred about the origin in the complex plane [6. This is the so called circle law [17, 3, which has recently been proved to remain true for general i.i.d. distributions [31. Another mathematical property proved true in the Gaussian case by explicit calculation [7] is that for large $N$ the expected number of real eigenvalues is asymptotically $\sqrt{2 N / \pi}$. Numerical evidence presented in [7] suggests this result persists for general i.i.d. distributions of mean zero and unit variance, although a proof is yet to be found. 
Of interest in both the physics and mathematics literature has been the integrability properties of eigenvalue distribution in the Gaussian case. Ginibre [16] was the first to seek an analytic formula for the eigenvalue probability density function (p.d.f.), giving rise to the name real Ginibre ensemble for real Gaussian matrices. In [16] an analytic expression was found for the eigenvalue p.d.f. conditioned so that all eigenvalues are real. For the conditioning specifying a general number of real eigenvalues, the analytic form of the eigenvalue p.d.f. was not obtained until the passing of a further twenty-five years [21, 6]. And it has not been until the last few years that analytic computations based on the eigenvalue p.d.f. have been mastered to the extent that the probability of a prescribed number of real eigenvalues can be calculated [20, 1, 26], and closed form expressions for the correlations obtained [11, 27, 15, 29, 10]. Furthermore, these analytic studies have been extended [12, 2] to the case of partially symmetric real Gaussian matrices [21].

A very recent result 28 relates to the average of the Schur polynomials $s_{\kappa}\left(\lambda_{1}, \ldots, \lambda_{N}\right)$ with respect to the eigenvalues $\left\{\lambda_{j}\right\}$ of matrices from the real Ginibre ensemble. We recall that the Schur polynomials are the basis for symmetric functions of $\left\{\lambda_{j}\right\}$, labelled by a partition $\kappa_{1} \geq \kappa_{2} \geq \cdots \geq \kappa_{N} \geq 0$ of non-negative integers, given by the ratio of determinants

$$
s_{\kappa}\left(\lambda_{1}, \ldots, \lambda_{N}\right)=\frac{\operatorname{det}\left[\lambda_{j}^{\kappa_{k}+N-k}\right]_{j, k=1, \ldots, N}}{\operatorname{det}\left[\lambda_{j}^{N-k}\right]_{j, k=1, \ldots, N}} .
$$

With $\left\{\lambda_{j}\right\}$ being the eigenvalues of the matrix $X$, the Schur polynomials are often written $s_{\kappa}(X)$. Making use of knowledge of the explicit form of the eigenvalue p.d.f., it is proved in [28] that for $X$ a member of the real Ginibre ensemble

$$
\left\langle s_{\kappa}(X)\right\rangle_{X}= \begin{cases}2^{|\kappa| / 2} \prod_{n=1}^{N} \frac{\Gamma\left(\left(N-n+\kappa_{n}+1\right) / 2\right)}{\Gamma((N-n+1) / 2}, & \text { all } \kappa_{n} \text { even } \\ 0, & \text { otherwise }\end{cases}
$$

where $|\kappa|:=\sum_{j=1}^{n} \kappa_{j}$.

It is the purpose of this paper to give a different viewpoint on this result. Explicitly, (1.3) will be deduced as a consequence of the theory of zonal polynomials. This viewpoint will allow for analogues of (1.3) to be given for the complex and real quaternion Ginibre ensemble. It further leads us to the evaluation of the moments of the characteristic polynomial in terms of generalized hypergeometric functions, in the case that the Gaussian matrices have a (matrix) distribution with a general variance. This in turn allows us to express the moments as different matrix integrals, in which the size of the matrix is equal to that of the moments, giving duality formulas. In the final section, again for the three Ginibre ensembles with a general variance, the averages of the power sums of the eigenvalues are expressed as finite sums of zonal polynomials.

\section{Zonal polynomials}

An alternative characterization of the Schur polynomials (1.3) is as the eigenfunctions of the differential operator

$$
\sum_{j=1}^{N}\left(\lambda_{j} \frac{\partial}{\partial \lambda_{j}}\right)^{2}+\frac{N-1}{\alpha} \sum_{j=1}^{N} \lambda_{j} \frac{\partial}{\partial \lambda_{j}}+\frac{2}{\alpha} \sum_{1 \leq j<k \leq N} \frac{\lambda_{j} \lambda_{k}}{\lambda_{j}-\lambda_{k}}\left(\frac{\partial}{\partial \lambda_{j}}-\frac{\partial}{\partial \lambda_{k}}\right)
$$


in the case $\alpha=1$, with the structure

$$
s_{\kappa}\left(\lambda_{1}, \ldots, \lambda_{N}\right)=m_{\kappa}+\sum_{\mu<\kappa} a_{\kappa \mu} m_{\mu} .
$$

In (2.2) $m_{\kappa}$ denotes the monomial symmetric function indexed by the partition $\kappa$ (e.g. with $N=2, m_{1^{2}}=$ $\left.\lambda_{1} \lambda_{2}+\lambda_{1} \lambda_{3}+\lambda_{2} \lambda_{3}\right), \mu<\kappa$ refers to the dominance ordering on partitions specifed by the requirement that $\sum_{j=1}^{l} \mu_{j} \leq \sum_{j=1}^{l} \kappa_{j}(l=1, \ldots, N)$, and the $a_{\kappa \mu}$ are scalars. For general $\alpha$ the eigenfunctions of (2.1) with the structure (2.2) are the symmetric Jack polynomials $P_{\kappa}^{(2 / \alpha)}\left(\lambda_{1}, \ldots, \lambda_{N}\right)$.

Associated with a partition $\kappa$ is the generalized Pochhammer symbol

$$
[u]_{\kappa}^{(\alpha)}=\prod_{j=1}^{N} \frac{\Gamma\left(u-(j-1) / \alpha+\kappa_{j}\right)}{\Gamma(u-(j-1) / \alpha)},
$$

and in terms of this and the classical Pochhammer symbol $(u)_{n}:=u(u+1) \cdots(u+n-1)$ one defines

$$
d_{\kappa}^{\prime}=\frac{\alpha^{|\kappa|}[(N-1) / \alpha+1]_{\kappa}^{(\alpha)}}{\bar{f}^{1 / \alpha}(\kappa)}, \quad \bar{f}^{1 / \alpha}(\kappa):=\prod_{1 \leq i<j \leq N} \frac{\left(1+(j-i-1) / \alpha+\kappa_{i}-\kappa_{j}\right)_{1 / \alpha}}{(1+(j-i-1) / \alpha)_{1 / \alpha}} .
$$

The quantity $d_{\kappa}^{\prime}$ in turn is used to define the renormalized Jack polynomials

$$
C_{\kappa}^{(\alpha)}\left(\lambda_{1}, \ldots, \lambda_{N}\right)=\frac{\alpha^{|\kappa|}|\kappa| !}{d_{\kappa}^{\prime}} P_{\kappa}^{(\alpha)}\left(\lambda_{1}, \ldots, \lambda_{N}\right)
$$

In the cases $\alpha=2,1$ and $1 / 2$ the renormalized Jack polynomials are the so called zonal polynomials associated with the symmetric spaces $g l(N, \mathbb{R}) / O(N), g l(N, \mathbb{C}) / U(N)$ and $u^{*}(2 N) / S p(2 N)[22$.

For present purposes, a key propery of the zonal polynomials is their appearance on the right hand sides of the matrix integrals [19, 22, 25.

$$
\begin{aligned}
\left\langle s_{\lambda}(A O)\right\rangle_{O \in O(N)} & = \begin{cases}\frac{C_{\kappa}^{(2)}\left(A A^{T}\right)}{C_{\kappa}^{(2)}\left((1)^{N}\right)}, & \lambda=2 \kappa \\
0, & \text { otherwise }\end{cases} \\
\left\langle s_{\lambda}(A U) s_{\kappa}\left(U^{\dagger} A^{\dagger}\right)\right\rangle_{U \in U(N)} & =\delta_{\lambda, \kappa} \frac{C_{\kappa}^{(1)}\left(A A^{\dagger}\right)}{C_{\kappa}^{(1)}\left((1)^{N}\right)} \\
\left\langle s_{\lambda}(A S)\right\rangle_{S \in S p(2 N)} & = \begin{cases}\frac{C_{\kappa}^{(1 / 2)}\left(A A^{\dagger}\right)}{C_{\kappa}^{(1 / 2)}\left((1)^{N}\right)}, & \lambda=\kappa^{2}, \\
0, & \text { otherwise }\end{cases}
\end{aligned}
$$

where in (2.6) the partition $2 \kappa$ is the partition obtained by doubling each part of $\kappa$, while in (2.8), $\kappa^{2}$ is the partition obtained by repeating each part of $\kappa$ twice. On the left hand sides the averages are over the classical groups $O(N), U(N), S p(2 N)$ of unitary matrices with real, complex and real quaternion elements respectively, endowered with the corresponding Haar measure.

We immediately observe a structual similarity between (1.3) and (2.6). In fact, as will be shown in the next section, (2.6) implies and furthermore generalizes (1.3). 


\section{Averages over the Ginibre ensembles}

That zonal polynomials are intimately related to averages over matrices with Gaussian entries is the theme of the monograph by Takemura [30. This theme has further been developed in the works [18, 25]. More generally, zonal polynomials can be related to any measure $d \mu(X)$ on the space of random matrices with real, complex or real quaternion entries possessing the property of being invariant under the mappings $X \mapsto U X, X \mapsto X U$ for $U \in O(N), U(N), S p(2 N)$ respectively. With $\langle\cdot\rangle_{X}$ denoting an average in such a setting, and $\langle\cdot\rangle_{U}$ denoting the average over the corresponding classical group, to apply this theory to the Ginibre ensembles we first make note of a fundamental factorization property of the zonal polynomials with respect to the former, namely (see e.g. 22])

$$
\left\langle C_{\kappa}^{(\alpha)}\left(A U^{\dagger} B U\right)\right\rangle_{U}=\frac{C_{\kappa}^{(\alpha)}\left(a_{1}, \ldots, a_{N}\right) C_{\kappa}^{(\alpha)}\left(b_{1}, \ldots, b_{N}\right)}{C_{\kappa}^{(\alpha)}\left((1)^{N}\right)} .
$$

Here $C_{\kappa}^{(\alpha)}\left((1)^{N}\right):=\left.C_{\kappa}^{(\alpha)}\left(x_{1}, \ldots, x_{N}\right)\right|_{x_{1}=\cdots=x_{N}=1}$ and $\left\{a_{i}\right\},\left\{b_{i}\right\}$ are the eigenvalues of $A, B$ respectively.

Proposition 1. [30] One has

$$
\left\langle C_{\kappa}^{(\alpha)}\left(A X B X^{\dagger}\right)\right\rangle_{X}=\frac{C_{\kappa}^{(\alpha)}(A) C_{\kappa}^{(\alpha)}(B)}{\left(C_{\kappa}^{(\alpha)}\left((1)^{N}\right)\right)^{2}}\left\langle C_{\kappa}^{(\alpha)}\left(X X^{\dagger}\right)\right\rangle_{X} .
$$

Proof. For any $f(X)$ integrable with respect to $d \mu(X)$, the invariance of $d \mu(X)$ under $X \mapsto U X$ tells us that

$$
\left\langle f\left(A X B X^{\dagger}\right)\right\rangle_{X}=\left\langle\left\langle f\left(A U X B X^{\dagger} U^{\dagger}\right)\right\rangle_{U}\right\rangle_{X}
$$

while the invariance under $X \mapsto X U$ gives

$$
\left\langle f\left(A X B X^{\dagger}\right)\right\rangle_{X}=\left\langle\left\langle f\left(A X U B U^{\dagger} X^{\dagger}\right)\right\rangle_{U}\right\rangle_{X}
$$

Choosing $f=C_{\kappa}^{(\alpha)}$ in (3.3) and using (3.1) shows

$$
\left\langle C_{\kappa}^{(\alpha)}\left(A X B X^{\dagger}\right)\right\rangle_{X}=\frac{C_{\kappa}^{(\alpha)}(A)}{C_{\kappa}^{(\alpha)}\left((1)^{N}\right)}\left\langle C_{\kappa}^{(\alpha)}\left(X B X^{\dagger}\right)\right\rangle_{X} .
$$

Choosing $f=C_{\kappa}^{(\alpha)}$ in (3.4) and again using (3.1) allows the right hand side of (3.5) to be evaluated, and (3.2) results.

We can use (3.2) combined with (2.6) (2.8) to obtain a generalization of (1.3) for each of the real, complex and real Ginibre ensembles. First we consider the case of a general measure $d \mu(X)$ invariant under multiplication by unitary matrices.

Proposition 2. Let $d \mu(X)$ be as required for (3.2). For real matrices

$$
\left\langle s_{\mu}(A X)\right\rangle_{X}= \begin{cases}\frac{C_{\kappa}^{(2)}\left(A A^{T}\right)}{\left(C_{\kappa}^{(2)}\left((1)^{N}\right)\right)^{2}}\left\langle C_{\kappa}^{(1)}\left(X X^{T}\right)\right\rangle_{X}, & \mu=2 \kappa \\ 0, & \text { otherwise; }\end{cases}
$$

for complex matrices

$$
\left\langle s_{\mu}(A X) s_{\kappa}\left(X^{\dagger} A^{\dagger}\right)\right\rangle_{X}=\delta_{\lambda, \kappa} \frac{C_{\kappa}^{(1)}\left(A A^{\dagger}\right)}{\left(C_{\kappa}^{(1)}\left((1)^{N}\right)\right)^{2}}\left\langle C_{\kappa}^{(1)}\left(X X^{\dagger}\right)\right\rangle_{X} ;
$$


for real quaternion matrices

$$
\left\langle s_{\mu}(A X)\right\rangle_{X}= \begin{cases}\frac{C_{\kappa}^{(1 / 2)}\left(A A^{T}\right)}{\left(C_{\kappa}^{(1 / 2)}\left((1)^{N}\right)\right)^{2}}\left\langle C_{\kappa}^{(1)}\left(X X^{\dagger}\right)\right\rangle_{X}, & \mu=\kappa^{2} \\ 0, & \text { otherwise. }\end{cases}
$$

Proof. Consider first (3.6). It follows from (2.6) that

$$
\begin{aligned}
\left\langle s_{\mu}(A X)\right\rangle_{X} & =\left\langle\left\langle s_{\mu}(A X O)\right\rangle_{O}\right\rangle_{X} \\
& = \begin{cases}\frac{\left\langle C_{\kappa}^{(2)}\left(A^{T} A X X^{T}\right)\right\rangle_{X}}{C_{\kappa}^{(2)}\left((1)^{N}\right)}, & \mu=\kappa^{2} \\
0, & \text { otherwise. }\end{cases}
\end{aligned}
$$

Making use of (3.2) in the case $\alpha=2$ gives (3.6). The derivation of (3.7) and (3.8) begins with (2.7) and (2.8), then makes use of (3.2) in an analogous manner.

We see from (3.6) and (2.3) that (1.3) is reclaimed if we can show that for $d \mu(X) \propto e^{-(1 / 2) \operatorname{Tr} X X^{T}}(d X)$,

$$
\frac{1}{C_{\kappa}^{(2)}\left((1)^{N}\right)}\left\langle C_{\kappa}^{(2)}\left(X X^{T}\right)\right\rangle_{X}=2^{|\kappa| / 2}[N / 2]_{\kappa}^{(2)} .
$$

For this we change variables $X X^{T}=A$, using the result $(d X) \propto(\operatorname{det} A)^{-1 / 2}(d A)$ (see e.g. [9, eq. (3.30)]), to obtain

$$
\left\langle C_{\kappa}^{(2)}\left(X X^{T}\right)\right\rangle_{X}=\frac{1}{C} \int_{A>0} e^{-(1 / 2) \operatorname{Tr} A}(\operatorname{det} A)^{-1 / 2} C_{\kappa}^{(2)}(A)(d A)
$$

where $C$ is such that the RHS equals unity when $\kappa=0^{N}$, and $A>0$ denotes that the integral is over the space of positive definite matrices. Changing variables now to the eigenvalues and eigenvectors of $A$ shows that (3.10) is proportional to

$$
\int_{0}^{\infty} d \lambda_{1} \ldots \int_{0}^{\infty} d \lambda_{N} \prod_{l=1}^{N} \lambda_{l}^{-1 / 2} e^{-\lambda_{l} / 2} C_{\kappa}^{(2)}\left(\lambda_{1}, \ldots, \lambda_{N}\right) \prod_{j<k}^{N}\left|\lambda_{k}-\lambda_{j}\right| .
$$

We recognize the integral in (3.11) as appearing in the integration formula (see [9, eq. (12.152)])

$$
\begin{aligned}
& \frac{1}{C} \int_{0}^{\infty} d \lambda_{1} \ldots \int_{0}^{\infty} d \lambda_{N} \prod_{l=1}^{N} \lambda_{l}^{a} e^{-\lambda_{l}} C_{\kappa}^{(\alpha)}\left(\lambda_{1}, \ldots, \lambda_{N}\right) \prod_{j<k}^{N}\left|\lambda_{k}-\lambda_{j}\right|^{2 / \alpha} \\
& \quad=C_{\kappa}^{(\alpha)}\left((1)^{N}\right)[a+(N-1) / \alpha+1]_{\kappa}^{(\alpha)}
\end{aligned}
$$

(take $\alpha=2, a=-1 / 2$ and change variables $t_{l} \mapsto t_{l} / 2$ ), which itself is a limiting case of a generalization of the Selberg integral conjectured by Macdonald and proved by Kadell and Kaneko (see [13] and references therein), and (3.9) follows.

Proceeding similarly, making use of (3.12) for $\alpha=1$, we can show that for $X$ complex, with $d \mu(X) \propto$ $e^{-\operatorname{Tr}\left(X X^{\dagger}\right)}(d X)$

$$
\frac{1}{C_{\kappa}^{(1)}\left((1)^{N}\right)}\left\langle C_{\kappa}^{(1)}\left(X X^{\dagger}\right)\right\rangle_{X}=[N]_{\kappa}^{(1)} .
$$

And for $X$ real quaternion with $d \mu(X) \propto e^{-\operatorname{Tr}\left(X X^{\dagger}\right)}(d X)$ - the trace now being with respect to the quaternion structure and so selecting only one of the diagonal elements from each $2 \times 2$ block - we have

$$
\frac{1}{C_{\kappa}^{(1 / 2)}\left((1)^{N}\right)}\left\langle C_{\kappa}^{(1 / 2)}\left(X X^{\dagger}\right)\right\rangle_{X}=2^{-|\kappa|}[2 N]_{\kappa}^{(2)}
$$


Substituting (3.10), (3.13) and (3.14) in Proposition 2 gives the sought generalization of (1.3) for the Ginibre ensembles.

Corollary 1. With the distribution of the real Ginibre ensemble proportional to $e^{-(1 / 2) \operatorname{Tr} X X^{T}}$, and the distribution of the complex and real quaternion ensembles proportional to $e^{-\operatorname{Tr}\left(X X^{\dagger}\right)}$, one has for the real Ginibre ensemble

$$
\left\langle s_{\mu}(A X)\right\rangle_{X}= \begin{cases}\frac{2^{|\kappa|}[N / 2]_{\kappa}^{(2)}}{C_{\kappa}^{(2)}\left((1)^{N}\right)} C_{\kappa}^{(2)}\left(A A^{T}\right), & \mu=2 \kappa \\ 0, & \text { otherwise }\end{cases}
$$

for the complex Ginibre ensemble

$$
\left\langle s_{\mu}(A X) s_{\kappa}\left(X^{\dagger} A^{\dagger}\right)\right\rangle_{X}=\delta_{\mu, \kappa} \frac{[N]_{\kappa}^{(1)}}{C_{\kappa}^{(1)}\left((1)^{N}\right)} C_{\kappa}^{(1)}\left(A A^{\dagger}\right) ;
$$

and for the real quaternion Ginibre ensemble

$$
\left\langle s_{\mu}(A X)\right\rangle_{X}= \begin{cases}\frac{2^{-|\kappa|}[2 N]_{\kappa}^{(1 / 2)}}{C_{\kappa}^{(1 / 2)}\left((1)^{N}\right)} C_{\kappa}^{(1 / 2)}\left(A A^{\dagger}\right), & \mu=\kappa^{2} \\ 0, & \text { otherwise. }\end{cases}
$$

The fact that [9, Prop. 12.23]

$$
P_{\kappa}^{(\alpha)}\left((1)^{N}\right)=\frac{\alpha^{|\kappa|}[N / \alpha]_{\kappa}^{(\alpha)}}{h_{\kappa}}
$$

where [9, Prop. 12.28] $1 / h_{\kappa}$ is the coefficient of $\left(x_{1}+\cdots+x_{N}\right)^{|\kappa|}$ in $P_{\kappa}^{(\alpha)}(x)$ allows the results of Corollary 1 to be written

$$
\begin{aligned}
& \left\langle s_{\mu}(A X)\right\rangle_{X}= \begin{cases}h_{\kappa} P_{\kappa}^{(2)}\left(A A^{T}\right), & \mu=2 \kappa \\
0, & \text { otherwise; }\end{cases} \\
& \left\langle s_{\mu}(A X) s_{\kappa}\left(X^{\dagger} A^{\dagger}\right)\right\rangle_{X}=\delta_{\mu, \kappa} h_{\kappa} P_{\kappa}^{(1 / 2)}\left(A A^{\dagger}\right) \\
& \left\langle s_{\mu}(A X)\right\rangle_{X}= \begin{cases}h_{\kappa} P_{\kappa}^{(1 / 2)}\left(A A^{\dagger}\right), & \mu=\kappa^{2} \\
0, & \text { otherwise. }\end{cases}
\end{aligned}
$$

respectively. In this form Corollary 1 appears in the unpublished manuscript [25] of one of us (EMR).

\section{Hypergeometric functions}

We know from workings in 4 that

$$
\begin{aligned}
\frac{2^{|\kappa|}[N / 2]_{\kappa}^{(2)}}{C_{\kappa}^{(2)}\left((1)^{N}\right)} & =\left.\frac{1}{|\kappa| ! 2^{|\kappa|}} d_{2 \kappa}^{\prime}\right|_{\alpha=1} \\
\frac{[N]_{\kappa}^{(1)}}{C_{\kappa}^{(1)}\left((1)^{N}\right)} & =\frac{1}{|\kappa| !}\left(\left.d_{\kappa}^{\prime}\right|_{\alpha=1}\right)^{2} \\
\frac{2^{-|\kappa|}[2 N]_{\kappa}^{(1 / 2)}}{C_{\kappa}^{(1 / 2)}\left((1)^{N}\right)} & =\left.\frac{1}{2^{|\kappa|}|\kappa| !} d_{\kappa^{2}}^{\prime}\right|_{\alpha=1},
\end{aligned}
$$


which we substitute into the results of Corollary 1 as appropriate. To see the consequence of this, we recall the definition of the generalized hypergeometric functions

$$
{ }_{p} F_{q}^{(\alpha)}\left(a_{1}, \ldots, a_{p}, b_{1}, \ldots, b_{q} ; x_{1}, \ldots, x_{N}\right):=\sum_{\kappa} \frac{1}{|\kappa| !} \frac{\left[a_{1}\right]_{\kappa}^{(\alpha)} \cdots\left[a_{p}\right]_{\kappa}^{(\alpha)}}{\left[b_{1}\right]_{\kappa}^{(\alpha)} \cdots\left[b_{q}\right]_{\kappa}^{(\alpha)}} C_{\kappa}^{(\alpha)}\left(x_{1}, \ldots, x_{N}\right) .
$$

For $p=0, q=1$ this is a generalization of the binomial expansion and we have [9, eq. (13.4)]

$$
{ }_{1} F_{0}^{(\alpha)}\left(a ; x_{1}, \ldots, x_{N}\right)=\prod_{j=1}^{N}\left(1-x_{j}\right)^{-a} .
$$

It follows from this that upon multiplying both sides of (3.15) and (3.17) by $[-r]_{\mu}^{(1)} /\left.d_{\mu}^{\prime}\right|_{\alpha=1}$, and both sides of (3.16) by $[-r]_{\mu}^{(1)}[-r]_{\kappa}^{(1)} /\left.\left.d_{\mu}^{\prime}\right|_{\alpha=1} d_{\kappa}^{\prime}\right|_{\alpha=1}$, and summing over $\mu$ (or $\mu$ and $\kappa$ in the case of (3.16)), the left hand sides of each of the identities can be summed according to (4.3). For the resulting matrix averages to be well defined, we require $r \in \mathbb{Z}_{\geq 0}$. To simplify the right hand sides, we note from (2.3) that

$$
[u]_{2 \kappa}^{(1)}=2^{2|\kappa|}[u / 2]_{\kappa}^{(2)}[(u+1) / 2]_{\kappa}^{(2)}, \quad[u]_{\kappa^{2}}^{(1)}=[u]_{\kappa}^{(1 / 2)}[u-1]_{\kappa}^{(1 / 2)},
$$

then make use of (4.2). Consequently we obtain the following set of matrix integral evaluations.

Corollary 2. Let $r \in \mathbb{Z}_{\geq 0}$. One has, for the real, complex and real quaternion Ginibre ensembles respectively,

$$
\begin{aligned}
& \left\langle\operatorname{det}\left(\mathbb{I}_{N}-A X\right)^{r}\right\rangle_{X}={ }_{2} F_{0}^{(2)}\left(-r / 2,(-r+1) / 2 ; 2 A A^{T}\right) \\
& \left\langle\operatorname{det}\left(\mathbb{I}_{N}-A X\right)^{r} \operatorname{det}\left(\mathbb{I}_{N}-X^{\dagger} A^{\dagger}\right)^{r}\right\rangle_{X}={ }_{2} F_{0}^{(1)}\left(-r,-r ; A A^{\dagger}\right) \\
& \left\langle\operatorname{det}\left(\mathbb{I}_{2 N}-A X\right)^{r}\right\rangle_{X}={ }_{2} F_{0}^{(1 / 2)}\left(-r,-r-1 ; A A^{\dagger} / 2\right) .
\end{aligned}
$$

Suppose $A$ in (4.4) is invertible, and write $\Sigma=\left(A^{\dagger} A\right)^{-1}$. We can then rewrite (4.4) to read

$$
\begin{aligned}
& \left\langle\operatorname{det}\left(\mathbb{I}_{N}-x X\right)^{r}\right\rangle_{X}={ }_{2} F_{0}^{(2)}\left(-r / 2,(-r+1) / 2 ; 2 x^{2} \Sigma\right) \\
& \left\langle\operatorname{det}\left(\mathbb{I}_{N}-x X\right)^{r} \operatorname{det}\left(\mathbb{I}_{N}-\bar{x} X^{\dagger}\right)^{r}\right\rangle_{X}={ }_{2} F_{0}^{(1)}\left(-r,-r ;|x|^{2} \Sigma\right) \\
& \left\langle\operatorname{det}\left(\mathbb{I}_{2 N}-x X\right)^{r}\right\rangle_{X}={ }_{2} F_{0}^{(1 / 2)}\left(-r,-r+1 ;|x|^{2} \Sigma / 2\right) .
\end{aligned}
$$

Here the averages are over $N \times N$ matrices $X$ with real, complex and real quaternion elements respectively having a distribution proportional to $e^{-\operatorname{Tr}\left(X X^{T} \Sigma^{-1}\right) / 2}$ in the real case and $e^{-\operatorname{Tr}\left(X X^{\dagger} \Sigma^{-1}\right)}$ in the complex and quaternion real cases. Introducing $Y=X-(1 / x) \mathbb{I}_{N}$ and setting $W=Y Y^{\dagger}$ we then have that $W$ is distributed as a non-central Wishart distribution (see e.g. 24]) in the first case, and its complex and real quaternion generalization in the other two cases. Furthermore, for $r=2 s$ even in the real case, and for general non-negative integer $r$ in the complex and real quaternion cases, the determinants in (4.5) can be written entirely in terms of $W$, and we obtain

$$
\begin{aligned}
& |x|^{2 N s}\left\langle(\operatorname{det} W)^{s}\right\rangle_{W}={ }_{2} F_{0}^{(2)}\left(-s,-s+1 / 2 ; 2 x^{2} \Sigma\right) \\
& |x|^{2 N r}\left\langle(\operatorname{det} W)^{r}\right\rangle_{W}={ }_{2} F_{0}^{(1)}\left(-r,-r ;|x|^{2} \Sigma\right) \\
& |x|^{N r}\left\langle(\operatorname{det} W)^{r / 2}\right\rangle_{W}={ }_{2} F_{0}^{(1 / 2)}\left(-r,-r-1 ;|x|^{2} \Sigma / 2\right) .
\end{aligned}
$$


The identities (4.6) are noteworthy for the fact that in the real case a different generalized hypergeometric function evaluation is known [24, Th. 10.3.7],

$$
\left\langle(\operatorname{det} W)^{s}\right\rangle_{W}=(\operatorname{det} \Sigma)^{s} 2^{N s} \frac{\Gamma_{m}((N / 2+s))}{\Gamma_{m}(N / 2)}{ }_{1} F_{1}^{(2)}\left(-s ; N / 2 ;-\Sigma^{-1} / 2 x^{2}\right)
$$

where

$$
\Gamma_{m}(u):=\pi^{m(m-1) / 2} \prod_{j=1}^{m} \Gamma(u-(i-1) / 2) .
$$

This implies (after some minor simplification) the identity between generalized hypergeometric functions

$$
{ }_{2} F_{0}^{(2)}(-s,-s+1 / 2 ; Y)=[N / 2]_{s^{N}}^{(2)}(\operatorname{det} Y)^{s}{ }_{1} F_{1}^{(2)}\left(-s ; N / 2 ;-Y^{-1}\right) .
$$

Using the property of Jack polynomials [9, Exercises 12.1 q.1 and 2]

$$
(\operatorname{det} Y)^{s} P_{\kappa}^{(\alpha)}\left(Y^{-1}\right)=P_{\kappa^{s}}^{(\alpha)}(Y)
$$

where $\kappa^{s}:=\left(s-\kappa_{N}, s-\kappa_{N-1}, \ldots, s-\kappa_{1}\right)$, noting from (2.4) that

$$
\frac{2^{|\kappa|}}{2^{\left|\kappa^{s}\right|}} \frac{d_{\kappa^{s}}^{\prime}}{d_{\kappa}^{\prime}}=\frac{[(N+1) / 2]_{\kappa^{s}}^{(2)}}{[(N+1) / 2]_{\kappa}^{(2)}}
$$

and using the property of the generalized Pochhammer symbol (2.3)

$$
[u]_{\kappa^{s}}^{(\alpha)}=(-1)^{\left|\kappa^{s}\right|} \frac{[(N-1) / \alpha-u+1-s]_{s^{N}}^{(\alpha)}}{[(N-1) / \alpha-u+1-s]_{\kappa}^{(\alpha)}}
$$

(a consequence of the functional equation for the gamma function), (4.8) can be verified directly by comparing coefficients of $P_{\kappa}^{(2)}(Y)$ on both sides.

\section{Duality identities}

There are many matrix ensembles of $N \times N$ matrices $\{X\}$ for which $\left\langle\operatorname{det}\left(\mathbb{I}_{N}-x X\right)^{r}\right\rangle_{X}$ can be expressed in terms of an average over dual matrix ensembles where the size of the matrices is $r \times r$ (see e.g. 9]). An example of relevance to the present study is an identity of Fyodorov and Khorozhenko [14] (see also [8]), which reads

$$
\begin{aligned}
& \left\langle\left|\operatorname{det}\left(z \mathbb{I}_{N}-A U\right)\right|^{2 p}\right\rangle_{U \in U(N)} \\
& \propto \int_{0}^{\infty} d t_{1} \cdots \int_{0}^{\infty} d t_{p} \prod_{l=1}^{p} \frac{\operatorname{det}\left(|z|^{2} \mathbb{I}_{p}+t_{l} A A^{\dagger}\right)}{\left(1+t_{l}\right)^{N+2 p}} \prod_{1 \leq j<k \leq p}\left|t_{k}-t_{j}\right|^{2} .
\end{aligned}
$$

To write the right hand side as a matrix average, we require a result from random matrix theory [9, Exercises 3.6 q.3] giving that the matrix $Y=X^{\dagger}\left(B^{-1 / 2}\right)^{\dagger} B^{-1 / 2} X$, where $X$ is an $(N+p) \times p$ standard complex Gaussian matrix, and $A$ is a complex Wishart matrix $B=b^{\dagger} b$ with $b$ and $(N+p) \times(N+p)$ standard complex Gaussian matrix, has eigenvalue p.d.f. proportional to

$$
\prod_{l=1}^{p} \frac{1}{\left(1+t_{l}\right)^{N+2 p}} \prod_{1 \leq j<k \leq p}\left|t_{k}-t_{j}\right|^{2} .
$$


Noting too that

$$
\prod_{l=1}^{p} \operatorname{det}\left(|z|^{2} \mathbb{I}_{p}+t_{l} A A^{\dagger}\right)=\operatorname{det}\left(|z|^{2} \mathbb{I}_{N p}+Y \otimes A A^{\dagger}\right)
$$

we see (5.1) can be rewritten as the matrix average duality

$$
\left\langle\left|\operatorname{det}\left(z \mathbb{I}_{N}-A U\right)\right|^{2 p}\right\rangle_{U \in U(N)}=\left\langle\operatorname{det}\left(|z|^{2} \mathbb{I}_{N p}+Y \otimes A A^{\dagger}\right)\right\rangle_{Y}
$$

The close relationship seen in $\$ 3$ between averages over the unitary group which contain an arbitrary matrix, and averages over the complex Ginibre ensemble, suggests analogous formulas hold for the averages in (4.5). This is indeed the case. The sought identities follow from a representation for ${ }_{2} F_{0}^{(\alpha)}(-r,-a / \alpha-(r-1) ; Y), r \in \mathbb{Z}^{+}$as an $r$-dimensional integral, valid for all $a>0$.

Proposition 3. Let $r \in \mathbb{Z}^{+}, a>0$ and $Y$ be an $N \times N$ matrix. We have

$$
\begin{aligned}
{ }_{2} F_{0}^{(\alpha)} & (-r,-a / \alpha-(r-1) ; Y) \\
& =\frac{1}{\widetilde{W}_{a-1,2 \alpha, r}} \int_{0}^{\infty} d t_{1} \cdots \int_{0}^{\infty} d t_{r} \prod_{l=1}^{r} e^{-t_{l}} t_{l}^{a-1} \operatorname{det}\left(\mathbb{I}_{N}+\left(t_{l} / \alpha\right) Y\right) \prod_{1 \leq j<k \leq r}\left|t_{k}-t_{j}\right|^{2 \alpha}
\end{aligned}
$$

where

$$
\widetilde{W}_{\lambda_{1}, \beta, n}:=\int_{0}^{\infty} d t_{1} \cdots \int_{0}^{\infty} d t_{n} \prod_{l=1}^{n} e^{-t_{l}} t_{l}^{\lambda_{1}} \prod_{1 \leq j<k \leq n}\left|t_{k}-t_{j}\right|^{\beta}
$$

(this normalization is a well known limiting case of the Selberg integral, and as such can be evaluated as a product of gamma functions [9, Prop. 4.7.3], although we don't need this fact).

Proof. With $\kappa^{\prime}$ denoting the conjugate partition, obtained by interchanging the rows and columns of the diagram of $\kappa$, we have [9, Exercises 12.4 q.2]

$$
[u]_{\kappa^{\prime}}^{(\alpha)}=(-\alpha)^{-|\kappa|}[-\alpha u]_{\kappa}^{(1 / \alpha)}
$$

Also, from the definition of $d_{\kappa}^{\prime}$ in terms of arm and leg lengths [9, Eq. (12.37)], and the corresponding definition of $h_{\kappa}$ [9. Eq. (12.58)], one sees

$$
d_{\kappa^{\prime}}^{\prime}=\left.\alpha^{|\kappa|} h_{\kappa}\right|_{\alpha \mapsto 1 / \alpha}
$$

Recalling (2.5) and (4.2) it follows

$$
{ }_{2} F_{0}^{(\alpha)}\left(-r,-a / \alpha-(r-1) ; x_{1}, \ldots, x_{N}\right)=\sum_{\kappa} \frac{\alpha^{-2|\kappa|}[r \alpha]_{\kappa}^{(1 / \alpha)}[a+(r-1) \alpha]_{\kappa}^{(1 / \alpha)}}{\left.h_{\kappa}\right|_{\alpha \mapsto 1 / \alpha}} P_{\kappa^{\prime}}^{(\alpha)}(X) .
$$

The significance of this series form, as distinct from the form implied by (4.2), is that the coefficient of $P_{\kappa^{\prime}}^{(\alpha)}(X)$ permits the integral representation

$$
\begin{aligned}
& \frac{\alpha^{-|\kappa|}[r \alpha]_{\kappa}^{(1 / \alpha)}[a+(r-1) \alpha]_{\kappa}^{(1 / \alpha)}}{\left.h_{\kappa}\right|_{\alpha \mapsto 1 / \alpha}} \\
& =\frac{1}{\widetilde{W}_{a-1,2 \alpha, r}} \int_{0}^{\infty} d t_{1} \cdots \int_{0}^{\infty} d t_{r} \prod_{l=1}^{r} t_{l}^{a-1} e^{-t_{l}} P_{\kappa}^{(1 / \alpha)}(T) \prod_{1 \leq j<k \leq r}\left|t_{k}-t_{j}\right|^{2 \alpha},
\end{aligned}
$$


which is just a rewrite of (3.12), after making use of (3.18). Substituting in (5.4), and recalling the dual Cauchy identity for Jack polynomials [9, Eq. (12.186)]

$$
\prod_{k, l=1}^{N}\left(1+x_{k} y_{l}\right)=\sum_{\kappa} P_{\kappa}^{(\alpha)}(X) P_{\kappa^{\prime}}^{(1 / \alpha)}(Y),
$$

(5.3) follows.

Corollary 3. Let the averages over $X$ be as in 4.5 . We have

$$
\begin{aligned}
& \left\langle\operatorname{det}\left(x \mathbb{I}_{N}-X\right)^{2 s}\right\rangle_{X} \\
& \quad=\frac{1}{\widetilde{W}_{0,4, r}} \int_{0}^{\infty} d t_{1} \cdots \int_{0}^{\infty} d t_{s} \prod_{l=1}^{s} e^{-t_{l}} \operatorname{det}\left(x^{2} \mathbb{I}_{N}+t_{l} \Sigma\right) \prod_{1 \leq j<k \leq s}\left(t_{k}-t_{j}\right)^{4} \\
& \left\langle\left|\operatorname{det}\left(x \mathbb{I}_{N}-X\right)\right|^{2 r}\right\rangle_{X} \\
& \quad=\frac{1}{\widetilde{W}_{0,2, r}} \int_{0}^{\infty} d t_{1} \cdots \int_{0}^{\infty} d t_{r} \prod_{l=1}^{r} e^{-t_{l}} \operatorname{det}\left(|x|^{2} \mathbb{I}_{N}+t_{l} \Sigma\right) \prod_{1 \leq j<k \leq r}\left(t_{k}-t_{j}\right)^{2} \\
& \left\langle\operatorname{det}\left(x \mathbb{I}_{2 N}-X\right)^{r}\right\rangle_{X} \\
& \quad=\frac{1}{\widetilde{W}_{0,1, r}} \int_{0}^{\infty} d t_{1} \cdots \int_{0}^{\infty} d t_{r} \prod_{l=1}^{r} e^{-t_{l}} \operatorname{det}\left(|x|^{2} \mathbb{I}_{2 N}+t_{l} \Sigma\right) \prod_{1 \leq j<k \leq r}\left|t_{k}-t_{j}\right| .
\end{aligned}
$$

Each of the right hand sides in the above identities can be written as matrix averages involving Wishart matrices. For example, with $Y=a^{\dagger} a$, where $a$ is an $r \times r$ matrix of standard complex Gaussian entries, (5.6) can be written

$$
\left\langle\left|\operatorname{det}\left(x \mathbb{I}_{N}-X\right)\right|^{2 r}\right\rangle_{X}=\left\langle\operatorname{det}\left(|x|^{2} \mathbb{I}_{2 N r}+Y \otimes \Sigma\right)\right\rangle_{Y} .
$$

In the theory of the complex Ginibre ensemble with $\Sigma=\mathbb{I}_{N}$ it is well known that the eigenvalue density $\rho_{(1)}((x, y))$ (with $N \mapsto N+1$ for convenience) satisfies

$$
\rho_{(1)}((x, y))=\frac{e^{-|z|^{2}}}{\pi N !}\left\langle\left|\operatorname{det}\left(z \mathbb{I}_{N}-X\right)\right|^{2}\right\rangle_{X}
$$

(see e.g. [14, eq. (1.3)]). Here $z=x+i y$ and $\{X\}$ is the complex Ginibre ensemble with $\sigma=\mathbb{I}_{N}$. Even though the equality is restricted to $\Sigma=\mathbb{I}_{N}$, this motivates us to use the duality identity (5.6) to investige the right hand side of (5.9) when $\{X\}$ is the complex Ginibre ensemble for a more general variance matrix. In particular, suppose $\Sigma=\operatorname{diag}\left(\sigma,(1)^{N-1}\right)$. Then (5.6) gives that the right hand side of (5.9) equals

$$
\frac{\sigma}{\pi N !} \int_{|z|^{2}}^{\infty} e^{-t} t^{N} d t+\frac{(1-\sigma)|z|^{2}}{\pi N !} \int_{|z|^{2}}^{\infty} e^{-t} t^{N-1} d t
$$

For $0<|z|^{2}<N$ and $N$ large the leading behaviour is

$$
\frac{\sigma}{\pi}+\frac{(1-\sigma)|z|^{2}}{\pi N}
$$

while for $|z|=\sqrt{N}-r$ and $\sigma$ fixed, for large $N$ we obtain

$$
\frac{1}{2 \pi}(1+\operatorname{erf}(\sqrt{2} r))
$$


independent of $\sigma$. Since the boundary of the eigenvalue support is $|z|=\sqrt{N}$, this suggests our choice of $\Sigma$ did not effect the largest eigenvalues. On the other hand, if we were to set $\sigma \mapsto \sigma \sqrt{N}$, as well as $|z|=\sqrt{N}-r$, the asymptotic form of (5.10) is dependent on $\sigma$, indicating that then the largest eigenvalues have been altered.

\section{Power sum averages}

In the previous section we computed, in (4.5), the integer moments of the characteristic polynomials for real, complex and real quaternion Gaussian matrices with a general variance matrix $\Sigma$. Here we average the power sums $p_{k}(X):=\operatorname{Tr}\left(X^{k}\right)$ over the same class of matrices. For real Gaussian matrices with $\Sigma=\mathbb{I}_{N}$, this average was computed using (3.15) in the case $A=\mathbb{I}_{N}$ 28. If we make use of each of the identities of Corollary 1 for general $A$, together with the expansion [22, Ex. 1.4.10]

$$
p_{k}(X)=\sum_{l=0}^{k-1}(-1)^{l} s_{\left(k-l, 1^{l}\right)}(X)
$$

we can generalize the result of 28 .

Corollary 4. Let the averages over $X$ be as in 4.5). We have

$$
\begin{aligned}
& \left\langle p_{k}(X)\right\rangle_{X}= \begin{cases}0, & k \text { odd } \\
2^{k / 2} \sum_{l=0}^{k / 2-1} \frac{[N / 2]_{\left(k / 2-l, 1^{l}\right)}^{(2)}}{C_{\left(k / 2-l, 1^{l}\right)}^{(2)}\left((1)^{N}\right)} C_{\left(k / 2-l, 1^{l}\right)}^{(2)}(\Sigma), & k \text { even }\end{cases} \\
& \left\langle p_{k}(X) p_{k}\left(X^{\dagger}\right)\right\rangle_{X}=\sum_{l=0}^{k-1} \frac{[N]_{\left(k-l, 1^{l}\right)}^{(1)}}{C_{\left(k-l, 1^{l}\right)}^{(1)}\left((1)^{N}\right)} C_{\left(k-l, 1^{l}\right)}^{(1)}(\Sigma) \\
& \left\langle p_{k}(X)\right\rangle_{X}= \begin{cases}0, & k \text { odd or } k / 2>N \\
2^{-k / 2} \frac{[2 N]_{1^{k / 2}}^{(1 / 2)}}{C_{1^{k / 2}}^{(1 / 2)}\left((1)^{N}\right)} C_{1^{k / 2}}^{(1 / 2)}(\Sigma), & k \leq 2 N \text { even. }\end{cases}
\end{aligned}
$$

Let $\rho_{(1)}^{\mathrm{r}}$ and $\rho_{(1)}^{\mathrm{c}}((x, y))$ denote the density of the real and (upper half plane) complex eigenvalues for real Gaussian matrices with variance matrix $\Sigma$. Then we have

$$
\left\langle p_{k}(X)\right\rangle_{X}=\int_{-\infty}^{\infty} x^{k} \rho_{(1)}^{\mathrm{r}}(x) d x+\int_{\mathbb{R}_{+}^{2}}\left((x+i y)^{k}+(x-i y)^{k}\right) \rho_{(1)}^{\mathrm{c}}((x, y)) d x d y
$$

where $\mathbb{R}_{+}^{2}:=\left\{(x, y): x \in \mathbb{R}, y \in \mathbb{R}^{+}\right\}$. The average in Corollary 4 in the complex case can be rewritten as an average involving both the one and two point correlations, which we refrain from writing down. In the real quaternion case, the average again can be written in terms of just the one point density. Here there are no real eigenvalues, and we have

$$
\left\langle p_{k}(X)\right\rangle_{X}=\int_{\mathbb{R}_{+}^{2}}\left((x+i y)^{k}+(x-i y)^{k}\right) \rho_{(1)}^{\mathrm{c}}((x, y)) d x d y .
$$

\section{Acknowledgements}

The work of PJF was supported by the Australian Research Council. 


\section{References}

[1] G. Akemann and E. Kanzieper, Integrable structure of Ginibre's ensemble of real random matrices and a Pfaffian integration theorem, J. Stat. Phys. 129 (2007), 1159-1231.

[2] G. Akemann, M.J. Phillips, and H.-J. Sommers, Characteristic polynomials in real Ginibre ensembles, J. Phys. A 42 (2009), 012001 (9pp).

[3] Z.D. Bai, Circular law, Ann. Prob. 25 (1997), 494-529.

[4] A. Borodin and P.J. Forrester, Increasing subsequences and the hard-to-soft transition in matrix ensembles, J.Phys. A 36 (2003), 2963-2981.

[5] A. Borodin and C.D. Sinclair, The Ginibre ensemble of real random matrices and its scaling limit, arXiv:0805.2986.

[6] A. Edelman, The probability that a random real Gaussian matrix has $k$ real eigenvalues, related distributions, and the circular law, J. Multivariate. Anal. 60 (1997), 203-232.

[7] A. Edelman, E. Kostlan, and M. Shub, How many eigenvalues of random matrix are real?, J. Amer. Math. Soc. 7 (1994), 247-267.

[8] Z.M. Feng and J.P. Song, Integrals over the circular ensembles relating to classical domains, J. Phys. A, to appear, 2009.

[9] P.J. Forrester, Log-gases and Random Matrices, Princeton University Press, to appear, 2009.

[10] P.J. Forrester and A. Mays, A method to calculate correlation functions for $\beta=1$ random matrices of odd size, J. Stat. Phys. 134 (2009), 443-462.

[11] P.J. Forrester and T. Nagao, Eigenvalue statistics of the real Ginibre ensemble, Phys. Rev. Lett. 99 (2007), 050603.

[12] P.J. Forrester and T. Nagao, Skew orthogonal polynomials and the partly symmetric real Ginibre ensemble, J. Phys. A 41 (2008), 375003 (19pp).

[13] P.J. Forrester and S.O. Warnaar, The importance of the Selberg integral, Bull. Am. Math. Soc. 45 (2008), 489-534.

[14] Y.V. Fyodorov and B.A. Khoruzhenko, On absolute moments of characteristic polynomials of a certain class of complex random matrices, Comm. Math. Phys. 273 (2007), 561-599.

[15] S. Gemam, The spectral radius of large random matrices, Ann. Prob. 14 (1986), 1318-1328.

[16] J. Ginibre, Statistical ensembles of complex, quaternion, and real matrices, J. Math. Phys. 6 (1965), 440-449.

[17] V. Girko, Circular law, Th. Prob. Appl. 29 (1984), 694-706.

[18] P.J. Hanlon, R.P. Stanley, and J.R. Stembridge, Some combinatorial aspects of the spectra of normally distributed random matrices, Contemp. Math. 138 (1992), 151-174.

[19] A.T. James, Distributions of matrix variate and latent roots derived from normal samples, Ann. Math. Statist. 35 (1964), 475-501. 
[20] E. Kanzieper and G. Akemann, Statistics of real eigenvalues in Ginibre's ensemble of random real matrices, Phys. Rev. Lett. 95 (2005), 230201.

[21] N. Lehmann and H.-J. Sommers, Eigenvalue statistics of random real matrices, Phys. Rev. Lett. 67 (1991), 941-944.

[22] I.G. Macdonald, Hall polynomials and symmetric functions, 2nd ed., Oxford University Press, Oxford, 1995.

[23] R.M. May, Will a large complex system be stable?, Nature 238 (1972), 413-424.

[24] R.J. Muirhead, Aspects of multivariate statistical theory, Wiley, New York, 1982.

[25] E.M. Rains, Attack of the zonal polynomials, Preprint, 1995.

[26] C.D. Sinclair, Averages over real Ginibre's ensemble of random real matrices, IMRN 2007 (2007), rnm015 (15pp).

[27] H.-J. Sommers, Symplectic structure of the real Ginibre ensemble, J. Phys. A 40 (2007), F671-F676.

[28] H.-J. Sommers and B.A. Khoruzhenko, Schur function averages for the real Gininbre ensemble, J. Phys. A 42 (2009), 222002 (8pp).

[29] H.-J. Sommers and W. Wieczorek, General eigenvalue correlations for the real Ginibre ensemble, J. Phys. A 41 (2008), 405003 (24pp).

[30] A. Takemura, Zonal polynomials, Institute of Mathematical Statistics, Hayward, CA, 1984.

[31] T. Tao and V. Vu, Random matrices: universality of ESPs and the circular law, arXiv:0807.4898, 2008. 